THE

MEDICO-PSYCHOLOGICAL ASSOCIATION.

\author{
THE COUNCIL, 1878-79.
}

PRESIDENT. -J. CRICHTON BROWNE, M.D.

PRESIDEAT RLECT. -J. A. LUSH, M.P., M.D.

EX-PRESIDENT.-G. FIELDING BLANDFORD, M.D.

TREABURER. JOHN H. PAUL, M.D.

xnitors or JOURXAL $\left\{\begin{array}{l}\text { T. \&. CLOUSTON, M.D. } \\ \text { HACK TUKE, M.D. } \\ \text { GEO. H. SA VAGE, M.D. }\end{array}\right.$

AUDTTORs. \{J. MORRAY LINDSAY, M.D.

\{. BOYD, M.D.

HON. BECRETARY YOR IRELAND,-E. M. COURTEMNAY, M.B.

HON. SECRETARY YOR BCOTLAND-J. RUTHERFORD, M.D.

GRNERAI 8TCRETARY, - BY. RAYNER, M.D.

JOSEPH LALOR, M.D.

T. L. ROGERS, M.D.

JOHN FRASER, M.B.

J. C. BUCKNILL, M.D.

WILLIAM ORAN̈GE, M.D.

T. HARRINGTON TÚ KE, M.D.

\section{Members of the Association.}

Adam, James, M.D. St. And., Medical Superintendent, Metrop. Dist. Asylum, Caterham, Surrey.

Adams, Josiah O., M.R.C.S. Eng., late Assistant Medical Officer, CitJ of London Asylum, Dartford; Brooke House, Clapton, London. Adams, Richard, L.R.C.P. Edin., M.R.C.S. Eng., Modical Superintendent, County

Aitken, Thomas, M.D. Edin., Medical Superintendent, District Asylum, Inverness. Aldridge, Charles, M.R.C.S., Plympton Bonse, Plympton, Deron.

Allen, T., M.D. St. And., M.R.C.S., Medical Superintendent of the Lanatio

Anderson, Thomas, M.B. Edin., M.R.C.P.E., Midlothian and Peobleo District Asylum, Rosewell, Edinburgh.

Ashe, Isaac, M.B., Medical Superintendent, Contral Criminal Asylum, Dandrum, Ireland.

Atkins, Ringrose, M.A., M.D. Queen's Univ. Ire., Med. Superintendent, District 2natic Asylum, Waterford.

Bacon, G. Mackenzie, M.D. St. And., M.R.C.S. Eng., Medical Superintendent, County Asylum Fulbourn, Cambridge.

Bailey, H Fredk. M. K C.S. Eng. L.S.A. . Nowport Pagnell.

Baillarger, M., M.D., Member of the Academy of Medicine, formerly Visiting Physician to the Salpétrière; 7, Rue de l'Université, Paris. (Hon. Mem.)

Baker, Benj. Ruseell, M.R.C.S., Bog., L.S A., Aseist. Med. Off. Preet wioh Aojlam, Manchester.

Baker, H. Morton, M.B. Edin. C.M. Weat Riding Anylum, Wakefield.

Baker, Robert, M.D., Edin., The Friends' Retreat, York.

Balfour, G. W., M.D. St. And., F.R.C.P. Edin., 17, Walter Street, Edinburgh.

Banks, Professor J T. A.B., M.D. Trin. Coll., Dub., F.K. and Q.C.P. Ireland, Visit. ing Physician, Kichmond District Asylum, il, Merrion Square East, Dublin.

Barton, Jas. Edwd., L R C.P. Fdin., L.M., M.R.C.S.. Ben. Asiat. Med. Officer, Surrey County Lunatio Asylum. Brookwood, Woting.

Bayley, J., M.R.C.S. Medical Superintendent, Lanatio Hospital, Northampton.

Beach, Fletcher, M.B., M.R.C.P. Lond., Medical Superintendent, Clapton Idiot Asylum. London.

Belgrave, T. B., M.D. Edin., 149, Hyde Part, Sydney, N.S.W., Australia.

Bell, John H.; M.D. Ed., M.R.C.S. Eng., Asst. Med. Officer, General Lunatio Hoopital, Northampton.

Benham, William T., M.D., C.M. Abdn., M.R.C.S. Eng., Med. Supt. Lunatio

Biff, M., M.D., Editor of the Italian "Journal of Mental Science," 16, Borgo di San Celeo, Milan. (Honorary Member.) 
ii.

Members of the Assocration.

Bigland, Thomas, M.R.C.S. Eng - L.S.A. Lond., Bigland Hall, Lancashire, and Medical Superintendent, The Priory, Roehampton.

Bishop, Sidney O., M.R.C.S. Eng., Negriting Upper Aseam, E. Indies.

Black, Cornelins. M.D. Lond.. M.: R.C.P , Winfir.l Manor, Derbyshire.

Blackall, John J Jeeph, M.D. Qu. Univ. and Killadysert, Co. Clare, Irel., late Assist. Med. Officer, Kichmond District Lunatic Asylum, Ireland.

Blake, John Aloysius, Esq., ex M.P., 12, Ely Place, Dublin., (Hon. Member.)

Blandford, George Fielding, M.D. Oxon, F.R.C.P. Lond., 71, Grosvenor Street, W. (PREsiderst, 1877).

Blatherwiok, T., M.R.C.S., Staff Surg.-Major, late Med. Oficer A rmy Lanat. Hosp., 1, St. Margaret's Banks, Rochester, Kent.

Bodington, George Fowler, M.D. Giessen, M.R.C.P. Lond., F.R.C.S. exam., Fing., Ashwood House Asylum, Kingswinford, Dndley, Staffordshire.

Boismont, M. Brierre de, M.D.. Member of the Academy of Medicine, 303, Rue du Faubourg St. Antoine, Paris. (Honorary Member.)

Bower, David, M.B. Aberd., Aøintant Phyrician, Saughton Hall Privato Asylum, near Edjinburgh

Bowes, John Ireland, M.R.C.8. Engl., L.S.A., Assint. Medical Superintendent, Noithampten County Anglum.

Boyd, Robert, M.D. Edin., F.R C.P. Lond, 1, Bolton Row, Mayfair, W. (PresiDENT, 1870 .

Braddon, Charles Hitchman, Esq., M.R.C.S. Eng., Mansefield, Cheotham Hill, Mancliester, Surgeon, County Gaol, Salford, Manchester.

Brodie David, M.D. St. And., L.R.C.S. Edin., Superintendent, Private Home for Imbeciles, Columbia Lodge, Liberton, Edinburgh.

Brown, Joseph John, M.B. Edin., Med. Supt. Fife and Kinross District Asylum, Cupar, Fife.

Brown, John Ansell, M R.C.8. Eng., L.S.A. Lond., late Medical Staff, Indian Army, Peckham House, Peckham.

Brown, William J., M.B. Assist. Med. Officer, Borough Asylum, Newcastle-on.Tyne.

Browne, William A. F., F.R.S.E., F.R.C.S.E., late Commissioner in Lunacy for Scotland ; Dumfries, N.B. (PREsiDeNT, 1866.) (Honorory Member.)

Browne, J. Crichton, M.D. Edin., F.R.S. Edin late Medical Superintendent, County Asylum, Wakefield, Lord Chancellor's Visitor, 45, Lincoln's Inn Fields. W.C. (Honorary Member.) (PR+RIDENT.)

Brushfield, Thomas Nadauld, M.D. St. And., M.R.C.S. Eng., Medical Superinterdent, County Asylum, Brookwood, Woking, Surrey.

Bucknill John Charles, M.D. Lond F.R.C.P. Lond F.R.S. J.P., late Lord Chancellor's Visitor ; 89, Wimpole street, London, W. Editor of Joumal, 1852-62. (PREsIDENT, 1860.) (Honorary Member, 1862-76.)

Buck, John, M.R.C.S., Medical Superintendent, Leicestershire and Rutland County Asslum, Leicester.

Burman, Wilkie, J., M.D. Edin., formerly Medical Superintendent, Wilts County Asylum, Devizes, Ogbourne St. George, near M. iriborng h.

Burrows Nir George, Bart., 18, Cavendish Square, London. W. (Honorary Member.)

Butler, John, M.D., the Retreat, Hartford, Connecticut, U.S. (Hon. Member.)

Byas, Edward, M.K.C.S. Eng., Grove Hall, Bow.

Cadell, Francis, M D. Edir.; 20, Castle Street, Edinburgh.

Cailleux, M. Girard de, M.D., Member of the Academy of Medicine, ex Inspec tor General of Asylums in the Prefecture of the Department of the Seine. (Honorary Member.)

Campbell, John A., M.D. Glas., Medical Superintendent, Cumberland and Westmoreland Asylum, Garlands, Carlisle.

Campbell, Donald C., M.D. Glas., M.R.C.P. Lond., F.R.C.P. Edin., Modical Superintendent, County Asylum, Brent wood, Essex.

Calmeil, M., M.D., Mernber of the Academy of Medicine, Paris, late Physician to the Asylum at Charenton, near Paris. (Honorary Member.)

Cameron, John, M.B., C.M. Edin., Medical Supt. Argyll and Bute Aeylum, Lochgilphead.

Cassidy, D. M., L.R.C.P.Edin, F.R.C.S.Edin , Med. Supt. Connty Asylum, Lancaster Chapman, Thomas Algernon, M.D. Glas., M.R.C.S. Edin., Hereford Co. and City Asylum, Hereford

Christie, Thomas B., M.D. St. And., F.R.S.E., F.R.C.P. Lond., F.R.C.P. Edin Medical Superintendent, Royal India Lunatic Asylum, Ealing, W. (Hon. General Secretary, 1872.)

Christison, Sir Robert, Bart., M.D. Edin., D.C.L. Oxf., late Professor of Materis Medica in the University of Edinuargh ; late Pres. R.S., Edin.; 40, Moray Place Edinburah. (Honorary Member.)

Claphsm, Wm Cruchley S., L R C:P M.R.C.S. Wt st Riding Asylum Wakefield.

Clapton, Edward, M.D. Lond., F.R.C.P. Lond., Physician, St. Thomas's Hospital, Visitor of Lunatics for Surrey; 10a, St. Thomas Street, Borougb.

Clarke, Lockhart, M.D., F.R.S., 21, New Cavendish Strott, W. (Bonorary Member.) 
Cleaton, John D., M.R.C.S. Eng., Commissioner in Lunacy, 19, Whitehall Place. (Honorary Member.)

Clouston, T. 8., M.D. Edin., F.R.C.P. Irdin., F.R.8.E., Phgsioian Superintendent, Rojal Aojlum, Morningeide, Edinburgh. (Editor of Journal.)

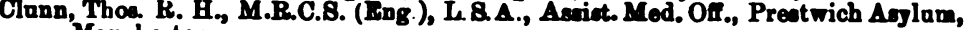
Manchester.

Cooke, Edwd. Marriott, M. B., M.R. C.S., Eng., Sen. Awiot, Med. Officer, City and Connty Asylum, Powick, Worcester.

Corley, $\triangle$ H., M.D. Queen's Univ., Ire., F.R.C.8.I. 30, Lower Bagot Street, Dublin. Courtenay, W. Masiere, A.B., M.B., C.M.T.C.D., Rosident Physician-Superintendent, District Hoopital for the Ineane, Limerick, Ireland. (Hon. Becretary
for Ireland.)

Craddook, Fredk. Hurst, B.A., M.R.C.8. Ing., L.S.A., St, Luke's Hoopital, Old Si reet E $\mathbf{0}$.

Cremonini, John, M.R.C.S. and L.8.A. Engl., Horton House, Hoxton, London, N.

Croudace, James H., L R C.S, Edin., S.M., L.S.A., Acoiot. Med. Officer Northnm. berland Asylum, Morreth.

Daniel, Jaoob, L R.C.P. and 8. Bd., W ye House, Buxton.

Daniel, W. C. M.D. Heidelb., M.R.C.8. Engl., Epeom, Burrey.

Davey, Jamee George, M.D. St. And., M.R.C.P. Lond., lato Medical Superintendent of the County Asylums, Hanwell and Colney Hatch, Middlear ; 4, Bodland Park Villas, Redland, Bristol.

Davideon, John H. M.D. Edin. Medical Baperintendent, County Asylum, Chester.

Davies, Francis P., M.B. Edin., M.R.C.S. Eng., Kent County Aoylum, Barming Heath, near Maidstone.

Davis, Robert A., M.D. St. And., L.R.C.P. Edin., Medical Superintendent, County Asylam, Burntwood, Lichfield.

Daxon, William, M.D. Queen's Univ., Ireland, F.R.C.S. Ireland, Reeident Physician, Ennis District Asylum, Ireland.

Deas, Peter Maury, M.B. \& M.S. Lond., Medical Superintendent, New Cheshire Asylum, Macclesfipid.

Delany, Barry, M.D. Queen's Univ., Ire., Med. Superintendent, District Aoylum, Kilkenny.

Delasiauve, M.. M.D., Member of the Academy of Medicine, Physician to the Bicêtre, Paris, 35, Rue des Mathurins-Saint-Jaoquea, Paris. (Hon. Member.)

Denholm, James, M.D., Dunse, N.B.

Denne, T. Vincent de, Esq., M.R.C.S. Eng., Aseistant Medical Superintendent General Lunatic Hospital, Northampton.

Déspine, Prosper, M.D., Rue du Loizir, Mareeilles. (Honorary Member.)

Dewsnap, William, M R.C.S. Eng., L.S.A., 1, Theresa Terrace, Hammersmith, W.

Dickson, F. K., F.R.C.P. Ed., Wye House'Lunatic Asylum, Buxton, Derbyshire.

Dickson, Hanmer, M. B. Edin., and C.M., 395, Nechello Park Road, Birmingham.

Down, J. Langdon Haydon, M.D. Lond., F.R.C.P. Lond., Physician to the London Hospital ; late Resident Physician Asylum for Idiote, Earlswood; 39, Welbeck St., Cavendish Sq., W., and Norman's Field Hampton Wiok.

Dore, W. Wateon, M.R.C.S. Eng., lete Assistant Medical Officer, County Asylum, Welle, Somerset, Alezandris, Egypt

Duckett, William Shaw, M.D. Glasgow, 44, Opper Mount Street, Dublin

Duncan, James Foulis, M.D. Trin. Col., Dub., F.K. and Q.C.P. Ireland, Visiting Physician, Farnham House, Finglas; 8, Upper Merrion Street, Dublin. (PResidret, 1875.)

Duncan Peter Martin, M.B. Lond., F.R.S., M.R.C.S. Ing., late Med. Super. Easez Hall Asylum; King'o Colloge, Btranó, W.C.

Dancan, J. Matthews, M.D., LL.D., F.R.C P. Ed., Phys. Aco. and Lecturer on Midwifery, \&o., St. Bartholomew's Hoopital, London, 71, Broot 8treet, Grosvenor Square, W.

Djer, Thomas B., M.D. Aberd., M.R.C.S. Eng., L.S.A., Aset. Medical Officor, County Asylum, Colney Hatch, Middleser.

Eager, Reginald, M.D. Lond., M.R.C.S. Eng. Northroode, near Bristol.

Eeger, Wilson, L.R.C.P. Lond., M.R.C.S. Eng., Mod. Superintendent, County Asylum, Meltnn, Suffolk.

Esmes, James A., M.D. St. And., F.R.C.S.I., Medical Superintendent, District Asylum, Cork.

Fastwood, J. William, M.D. Edin., M.R.C.P. Lond., Dinsdale Park, Darlington.

Edmundson, Joseph, M.D. St. And., L.K. and Q.C.P. Ire., M.R.C.S. Eng., District Asylum. Enniscorthy, Wexford, Ireland.

Flliot, G. Stanley, M.R.C.P. Ed., U R.C S. Ed., Assistant Medical Officer, County Asplum, Colney Hatch, Middlesex.

Eustace, J ,M.D. Trin. Col., Dub., L.R.O.S. Ireland ; Highfield, Drumcondra, Dublin. Eustace, Marcus, F.K.Q.C.P. Ire., Highfield, Drumcondra, Ireland.

Evans, Eran, M R.C.8. Bug., Mungter House, Fulham, Middlesex.

Fairless, William Dean, M.D. St. And., M.R.C.S. Eng., late Medical Superintendent, Old Royal Áglum, Montrose ; Kirklands, Bothwell, Lanarkshire, N.B. 
iv.

Members of the Association.

Falret, Jules, M.D., 114, Rue du Bac, Paris. (Honorary Member.) Farr, William, M D., Registrar General's Ottice, Somerset House. (Hm. Member.) Finch, W. Corbin, M.R.C.S. Eng., Fisherton House, Salisbury.

Finch, John E. M. M. M., Medical Superintendent, Bornugh Asylum, Leicester.

Fitzpat rick, T., M.D.Ed., F.K. and Q.C.P.. Ireland, 31 Lower Bagot Street, Dablin.

Flemming, C. F., M.D., Editor of the "Zeitgchrift fur Psychiatrie," late of the Sachrenberg State Asylum, Schwerin, Mecklenburgh. (Honorary Member.)

Fletcher, Robert V., Esq., L.R.C.S.I., L.R.C.P. and L.R.C.S. Ed., Medical Superin. tendent, District Asylum, Ballinasloe, Ireland.

Foville, Achille, M.D, Ruven, France. (Honorary Member.)

Fox, Edwin ('hurchill' Pigott, M.B. and M.C. t.din., Brislington, Bristol.

Fox, Francis Ker, M.D. Cantab, Brislington House, Bristol

Fox, Charles H., M.D. St. And., M.R.C.S. Eng., Brislington Honse, Bristol.

Fraser, John. M.B., C.M., Deputy Commisiuner in Lunaoy for Sootland, 31, Rexent Terrace, Edinburgh

Gairdner, W.T., M.D. Edin., Professor of Practice of Physic, 225, St. Vincent St.,

Gardiner Gideon G., M.D. St. And., M.R.C.S. Eng., 40, Finsbury Cirons, E.C.

Girner, W. H., Esq., F.R.C.S.I., A.B.T.C.D., Medical Superintendent, Clonmel District Asylum.

Gaquet, J. R., M.B. Lond., St. George's Retreat, Burgess Hill, and 127, Eastern Road, Briphton.

Gelston, R.' P., Esq., L.K. and Q.C.P.I., L.R.C.S.I., Assistant Medical Officer, Clonmel District Hospital for the Insane.

Gilohrist, James, M.D. Edin., Resident Physician, Crichton Royal Institution, Dumfries.

Gill, H. C., M.R.C.S. Eng., L.S.A., Medical Supt., The Lunatic Hoepital, York.

Gill, Stanley A.. M R (.P. Edin., M.K C.S. Eng., Med. Superint. KuJal Lunatic Aeylum. Liverponl.

Gilland, Robert B., M.D. Glas., L.F.P.S. Glas., M.R.C.S. Eng., L.S.A., Medical Superintendent, Berks County Asyjum, Moulsford, Wallingford.

Glendinniog. Jmes. M.D. Glas. L.R.C.S. Edin., I.M., Assiot. Med. Off. Joint Coun ies Aaylam, Abergareuny.

Gower, Rohert Munday, M.R.C.P. Lond. Senier Medical Officer, Millbank, London. Grabhanı, G. W. M.D. Lond., Res. Physician, Earlswood Asylum, Redhill, Surrey. Gray, John P. M.D. LL.D, Medical Superintendent, Stato Lunatio Agylum, Utica, New York. 'Honorary Member.)

Grieve, R, M.D. Medical Superintendent, Public Asylum, British Guians, Berbice. Green, T., M.R C.S. Eng., Medical Superintendent, Borough Asylum Birmingham. Greene, Richard, I.R.C.P. Edin., As. Med. Superint. L. Riding Asylum, Beverley. Grierson, S., M.R.C.S., Medical Superintendent, Border Counties Asylum,

Grifin, L. T., Esq., L.R.C.S.I., Ext. L.R.C.P. Lond., Visiting Physician, Killarney District Hospital for the Insane, Ireland, High Stret, Killarney, Cu. Kerry.

Guy, W. A., M.B. Cantab, late Prufessor of 'Hygiene, King's College, London (Homorary M ember), 12, Gordon Street, W.C.

Hall, Edward Thomas, M.R.C.S. Eng. Blackland s House Asylum, Chelsea.

Harbinson, Alexander, M.D. Ire., M.R.C.S. Eng., Assist. Med. Officer, County

Asylum, Lancaster.
Harmer, W. M, Esq., M.R.C.P. E., North Grove House Asylum, Hawkhurst, Kent. Harper, Henry Lewis, M.D. St. And., M.R.C.S. Eng., late Medical Superintendent, County Asylum, Chester ; 19, Addison Road, Kensington.

Hatchell, George W., M.D. Glas., L.K. and Q.C.P. Ireland, Inspector and Commissioner of Control of Asylums, Ireland, 16, Elgin Road, Dublin. (Hon. Mem.)

Hatchell, Jnseph H., L.K.Q.C.P.' Ire.. Resident Mod. Superintendent, Distriot Lanatic Asylum, Maryborough, Ireland.

Haughton, Rev. Profersor S., School of Physic. Trinity Coll., Dublin, M.D., T.C.D., D.C L. Oxnn, F.R.S. (Hon. Member)

Haynes, Stanley, M.D. Edin., Eastnor Honse. Malvern, Worcestershire.

Hearder, George J., M.D. St. And., L.R.C.S. Edin., Medical Superintendent, Joint Counties Asylum, Carmarthen.

Hemming, John Lamond, Esq., L.R.C.P. Lond., Munster House, Fulham, S.W

Hewson, John Dale. Esq., Ext. L.R.C.P. Eng., Medical Superintendent, Coton Hill Asylum, Stafford.

Hewson, R. W., L.R.C.P. Ed., Assist. Med. Officer, Royal Asylum, Cheadle, Manchester.

Hills, William Charles, M.D. Aber., M.R.C.S. Eng., Medical Superintendent, Norfolk County Asylum, Norwich.

Hingston, J. Tregelles, Esq., M.R.C.S., Eng., Medical Superintendent, North Riding Asylum, Clifton, York.

Hitohcock, Charles, L.R.C.P. Edin., M.R.C.S. Eng. Fiddington House, Market Lavington, Wilts.

Hitchman, J., M.D. Ft. And., F.R.C.P. Lond., F.R.C.S. Eng., late Medical Superintendent, County Asylum, Derby ; The Laurele, Fairford. (PursidrNT, 1856.) 
Hogg, Richard Bowren, M.R.O.S., L.S.1., Assist. Med. Offoer, Kent Coonty Asplum, Barming Heatli.

Holland, Joseph, F.R C.S. Rng., L.S.A., late Medical Superintendent, County Anylum, Whittingham, Preston

Howden, James C., M.D. Edin., Medical Superintendent, Montrose Royal Lunatio Asylum, Sunn yside, Montrose.

Bosking, kithelbert, M.R.C.S. Ei g., ILS.A., Aesist. Med. Officer Surrey Connty Aeplum, Tooting, 8.W.

Hngeard, William R., B.A.. M.D. Ou. Triv., Irel., C. M., Leytonstone. Faser.

Hullah, Robert, M.R.C.S., Surg. Supt. Eastern Prov. Lun. Asyl., Graham's Town,

Cape of Good Hope.
Humphry, John, M.R.C.S. Eng., Medical Superintendent, County Asylum, $\Delta$ yleabury, Bucks.

Hateon, E., M.D. Ed., Medical Buperintendent, Lunatio Anylum, Barbadoes. Iles, Daniel, M.R.C.S. Fng., Resident Modical Officer, Fairford House Rotreat,

Incels, Dr., Hoepice Gaislain a Ghent, Belginm.

Inglis, Thoma, F.R.C.P. Rdin., Acoietant Phycician, Bojal Anjlum, Morningside,

Irelend, W. W., M.D. Bdin., Medical Superintendont, Larbert Inotitution, Stirlingshire.

Isasa, J. B., M.D. Queen's Unir., Irel., Acoirt. Mod. Offoer, Brosdmoor, nr. Wokingham.

Jectson, J. Hughlings, M.D. St. And., F.R.O.P. Lond., Phyrician to the Hospital for Epilepey and Paralysis, do.; 3, Manchester Square, London, W.

Jackoon, J. J., M.R.C.S Fng. Medioal Buperintendent Lanatio Asylum, Jereer.

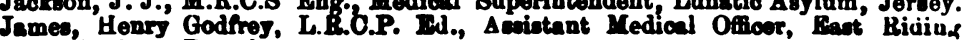
Aojlum, Beverlev.

Jamicenon, Robert, M.D. Edin., L.R.C.S. Irdin., Medical Superintendent, Royal Aoylum, Aberdeen.

Jarvis, Edward, M.D., Dorchester, Mass., D.S. (Honorary Member.)

Jepeon, Octarius, M.D. St. And., M.R.C.S. Eng., late Medical Superintendent, St. Lake's Hospital; Medical Superintendent, City of London Aerlum, Dartford.

Johnson, John, M.D. Edin., Belmont, Church-road, Tunbridge Wells.

Johnstone, J. Cariyle, M.B., C.M., Assist. Med. Oficer, Fite and Kinrose Asylum, Cupir, N.B.

Jones, Evan, M.R.C.S. Eng., Ty-mawr, Aberdare, Glamorganshire.

Kebbell, William, L.R.C.P. Lond., M.R.C.S. Eng., Asoistant Medical Officor, Three Counties Anylum, Stotfold, Baldock, Herte.

Kenyon, John Kilshaw, M.R.C.S., Fng., L.S.A., The Old Hall, Billingborongh, Lincoln shire.

Kirkbride, T. S.M.D. Phrsician-in Chief and Superintendent, Pennsylvania Hos. pital for the Insane, Philadelphia. (Honorary Memb-r.)

Kirkman, John, M.D., 1, Buckingham Road, Brighton. PkesiderT, 1862.

Kirkman, W. Phillips, M.D. St. And., M.B.C.S. Eng., L.S.A., The Briarn, St. Loonards-on-sea.

Kitching, Walter, M.R.C.8. Ingl., Heworth, York.

Griekenbeek C.A. M.D., Med Superint. Govt. Lanat. Asplum, Colombo, Ceylon.

Laehr, H., M.D., Sohweiser Hof, bei Berlin, Editor of the "Zoitechrift für Psychiatrie." (Honorary Member.)

Lalor, Joseph, M.D. Glas., L.R.C.S. Ireland, Resident Phyrician-Superintendent, Richmond District Aoglum, Dablin. (PresIDENT, 1861.)

Lewlees, Edmund, Esq., B.N., M.R.C.S. Eng., L.A.H. Dub., Medical Superintendent of St. Patrick's Hospital, Dublin.

Lawrence James Dr. Connty Arlum, Chester

Lasegue, M., M.D., Paris, Physician to the Neckar Hospital. (Honorary Member.) Leeper, Wm. Waugh, M.D. Ed., Loughgall, Co. Armagh.

Leidesdorf, M., M.D., Universität, Vienna. (Honorary Member.)

Levinge, Edward G., M.B. Dub., L.R.C.S. Ire., Aevist. Med. Officor Lanst. Aojlum, :tapleton Briatol.

Lowis, Henry, M.D. Bruss., M.R.C.S. Eng., L.S.A., late Assistant Medical Officer, County Asylum, Chester ; Weet Terrace, Folkestone, Kent.

Ley, H. Rooke, M.R.C.S. Eng., Modical Superintendent, County Asylum,

Liddell, W. A., M.D., Modical Superintendent Govan Paroohial Asylum, Glasgow.

Lindsa, William Iauder, M.D., F.R.S. Edin., F.L.S. Lond., Phyeician to the Murray Royal Institution, Perth ; Gilgal, Perth.

Lindsay, James Uurray, M.D. St. And, L. R.C.S. Edin., Modical Superintendent, County Asylum, Mickleover, Derbyshire.

Lister, Edward, L.R.C.P. Edin., M.B.C.S. Fng., Hagdook Lodge Retreat, Newton-le-Willows, Lanceghire.

Long, C. F., M.R.C.S. Fng., L.S.A., Medical Superintendent of Borought

Lovell, W. Day, L.R.C.P. Edin., M.R.C.S. Eng., L.S.A., Bradford.on·Arou, wesr Buth. 
ท.

Members of the Association.

Lovett, Henry A., M.R.C.S., Worcester Co. and City Lunstic Asylum, Powick, Worcester.

Lowe, William H., M.D. Edin., F.R.C.P. Edin., Woodcote Lodge, Inner Lodge, Wimbledun.

Lowry, Thomas Ha

itone Kent.

Lash, John Alfred, M.P., F. (President El Ect.) Lush, Wm. John Henry, F R C.P. Edin , L.M., M.R.C.S. Eng, F.L S., Fytield

Lyle, Thos., M.D. Glas., Assistant Physicisn, Rorongh Asylum, Birminghsm. Q.C.P. Ireland, M.R.C.S. Engl., Blackrock, Cork, Ireland. (Hon. Secretary

McDowall, T. W., M.D. Edin., L.R.C.S.E , Medical Superintendent, Northumberland County ARylum, Morpeth.

Mackenzie, Geo. H., M.B., Assist. Med. Officor, Glonoester County Lunatic Asylum.

Mackensie, Geo. Hald M.D. Durham and Glas., L.F.P.S. Glas., 10, Lancaster Road, Belsize Park, N. W.

Mackintosh Alerander, M.D. St. And, L.F.P.S. Glas., late Physician to Boyal Asylum, Gartnavel, Glasgow, 26, Wondside Place, Glasgow.

Maclaren, James, L.R.C.S.E., Stirling District Asylum, Larbert, N.B. Macleod, M.D. M.B., Assistant Medical Sapo

Maclood, William, M.D. Edin., Deputy Inspector-General, Naval Lunatic Hospital, Great Yarmonth.

Maclintoct John Rort, M.D. Aber., late Assistant Physician, Murray's Royal Inock, John Robert, M.D. Aber.,

Maconchy, John, M.B., T.C.D., F.R.C.S. Ireland, M.R.C.S. Engl., Infirmary House, Downpatrick.

Macmun, John, M.D. Glas., L.K. and Q.C.P. Ireland, L.F.P.S. Glas., Resident Physician, District Hospital for the Insane, Sligo.

M. W. Edin., L.M.Edin., Medical Superintendent, County Asylum, Wells, Somerset.

Major, Herbert, M.D. Med. Super.nt., West Riding Asyl., Wakefield.

Manley, John, M.D. Edin., M.R.r.S.

Asylum, Knowle, Fareham, Hants. for Gladesville, near Sydney.

A.C.S. Laverstock Houge, Salisburz.

Manning, Harry, B.A. London, M.R.C.S., S.A., Assistant Medical Officer, County

Marsh, Ww. Joseph, M.R.C.S. Eng., L.S.A., late Assistant Medical Oficer, County , W. Joseph, M.R.C.S. Eng., S. Shrewaburp.

Marshall, William G., M.R.C.S., MedicalSuperintendent, County Asylum, Colney

Hatch, Middlesex. University College, formerly Medical Superintendent, Royal Lunatic Hospita' University College, formerly Medicandon, W. (Editor of Journal, 1862-78.) (PrEsidereT, 1871.)

Merson, John, M.D. Aberd., Senior Assistant Medical Officer, West Riding Asylum, Wakefield.

MoIntosh, W.C., M.D. F.R.S. Ed., Med. Supt., Perth D.A.A., 14, Lower Pembroke

McDonnell, Robert,

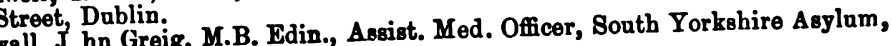
Wadsley.

M'Cullough, David M., M.D. Edin., Medical Superintendent mouth, Hereford, Brecon, and Radnor; A.P. Ireland, and L.R.C.S. Ireland,

M'Kinstry, Robert, M.D. Giess., L.K. and Q.C.P. Ireland, and L. formerly Physician, Trongh Fever Hospital and Gragh. Dispensaries : Resident Physician, District Asylum, Armagh.

Merrick, A.S., M.D. Qu. Oni. Irel., L. District Asslum, Belfast, Ireland. University of Göttingen.

Meyer, Ludwig, M.D. (Honorary Mrember.) Universit Hall Asylum, Bow.

Mickle, Julius, M.D., Medical Superintendent, Grove Hall Asylum, Bow. Lut. Luke' Mickley, George, M.A., M.B. Cantab.

Millar John, Esq., L.R.C.P. Edin., L.R.C.S. Edin., Late Medical Superintendent, County Asylum, Bucks ; Bethnal House, Cambridge Heath, London, Kolum,

Millson, George L.R C.P. Lond., M.R.C.S. Eng., Northampton County
Berrywood, Northampton.

Minchin, Humphry, A.B and M.B., T.C.D., F.R.C.S.T.
Dublin Prisons, 56, Lower Dominick Btreet, Dublin. 
Mitchell, Arthur, M.D. Aberd., LL.D., Commissioner in Lanaoy for Scotland ; 34, Drummond Place, Edinburgh. (Honorary Member.)

Mitchell, S., M.D. Edin., Medical Superintendent, Sonth Yorkshire Asylum, Wadsley, near Sheffield.

Moody, James M., M.R.C... Eng., Assist. Med. Offoer Surrey County Aoylum, Brookwood.'Woking.

Monro, Henry, M.D. Oron, F.R.C.P. Lond., Censor, 1861, Visiting Physioian, St. Luke's Hospital 13, Cavendish Square, London, W. (PrEsIDENT, 1864.)

Morean. M. (de Tours), M.D., Member of the Academy of Medicine, Senior Physician to the Saltpêtriere. Paris (Honorary Merıber.)

Mould, George W., M.R.C.S. Eng., Medical Superintendent, Royal Lunatio Hospital, Cheadle, Manchester.

Mairhead. Claud. M.D., F.R.C.P. 7, Heriot Row. Edinburgh

Mundy, Baron Jaromir, M.D. Würzburg, Profewor of Military Hygiene, Universität, Vienna. (Honorary Member.)

Munro, A. C., M,B. Edin., Asst. Med. Officer, Southern Counties Asylum, Dumfries.

Murray, Henry G., L.K Q C.P. (Irel ), L. H., L.R.C.S.I., Asoist. Med. Off. Prest-

Nairne, Robert, M.D. Cantab., F.R.C.P. Lond., late Senior Physician to St. George's Hospital, Commissioner in Lunacy ; 19, Whitehall Place, London. (Honorary Member.)

Noedham, Frederick, M.D. St. And., M.R.C.P. Edin., M.R.C.S. Eng., late Medical Superintendent, Hospital for the Insane, Bootham, York; Barnwood Honse, Gloucester.

Nowcombe, Charles Frederiok, M.B. Aberd., Asoistant.Medical Officor, County Lunatic Asjlum, Rainbill, Lancashiro.

Newington, Alezander, M.B. Camb., M.R.C.8. Eng., Wheatley, Oxon.

Newington, H. Hayes, M.R.C.S., Ticehurst, Sussex.

Nowington, Samuel, M.A. Oxon., M.R.C.P. Lond., Ridgway, Ticehurst, Sussex.

Nicholson, William Norris, Esq., 45, Lincoln's-Inn-Fields, W.C., Lord Chancellor's Visitor of Lunatics. (Honorary Member.)

Nicolson, David, M.B. and C.M. Aber., late Med. Off., H. M. Convict Prison, Ports mouth. Deputy Super, State Asylum, Broadmoor, Wokingham, Berks.

Niven, William, M.D. St. And., Medical Staff H.M. Indian Army, late Superintendent of the Government Lunatic Asylum, Bombay, care of Thacker, Vining and Co., Bombay.

North, S. W., Esq., M.R.C.S. E., F.G.S., Medical Attendant, Dannington House Asylum, Castlegate, York.

Nugent, John, M.B. Trin. Col., Dub., L.R.C.S. Ireland, Senior Inspector and Commissioner of Control of Asylams, Ireland ; 14, kutland Square, Dublin. (Honorary M (Ember.)

Ogle, John W., M.D. Oron., F.R.C.P. Lond., 30, Cavendish Square, London, W.

Orange, William, M.D. Heidelberg, M.R.C.P. Lond., Medical Superintendent, State Anylum, Broadmoor, Wokingham Kent.

Owen, Harold, M.R.C.S, Eng., L.R.C.P. Ed., Resident Medical Proprietor, TueBrook Villa Asylum, Liverpool.

Paley, Edward, M.D. M.R.C.S. Eng., late Ros. Medical Officer, Camberwell Honse, Camberwell ; Med. Superintendent, Yarra Bend Asy., Melbourne, Victoria.

Palmer, Edward, M.D. St. And., M.R.C.P. Lond., M.R.C.S., Medical Superin-

tendent, County Asylum, Lincoln.
Parsey, William Henry, M.D. Lond., M.A. Lond., M.R.C.P. Lond., Medical Superintendent, County Asylum, Hatton, Warwickshire. (President, 1876.)

Pater, W. Thompson, M R.C.S. Eng,, L.S.A., Modical Superintendent, County Lunatic Asylum, Stafford.

Paterson, G. A., M.D. Edin., F.R.C.P. Edin., late Depaty Commissioner in Lanacy; 15, Merchiston Pariz, Edinburgh.

Patton, Alex., M.B., Resident Medical Superintendent, Farnham House, Finglas, Co. Dublin.

Paul, John Hayball, M.D. St. And., M.R.C.P. Lond., F.R.C.P. Edin.; Camberwell House, Camberwell. (Treasurer.)

Peddie, Alerander, M.D. Edin., 15, Rutland Street, Edinburgh

Pedler, George H., L.R.C.P. Lond., II.R.C.S. Eng., 6, Trevor Terrace, Knights. bridge, $S$. W

Perkins, Whi:field, M.R.C.S. Eng., L.S.A., Asst. Medical Officer, City of London Lunatio Asylum, Stone, Dartford, Kent.

Petit, Joseph, L.R.C.S. Ire., L.R.C.S I., District Lunatio Asylum, Letterkenny. Pbilip, Jas. A., M.A., M.B.\& C.M. Aberd., Mavisbank, Lasswade, near E.linbargh. Philippe, Sutherland Rees, M.D., Qu. Uuiv.. Irel., C.M., F R.G.S., Langham Hotel, Londun.

Philipaon, George Hare, M.D. and M.A.Cantab., F.R.C.P. Lond., 7, Eldon Square, Newcastle.on-Tyne.

Pim, F., Esq., M.R.C.S. Eng., L.K. and Q.C.P. Ireland, Medical Superintendent Lucan Institution for the Insane and for Idiots, Lucan, Ireland.

Pitman, Henry A., M.D. Cantab., F.R.C.P. Lond., 28, Gordon Square, W.O., Registrar of Rojal College of Physicians. (Honorary Member.) 
viii.

Members of the Association.

Platt. - M. B. (Addresn uncommunicated).

Plaxton, Joseph Wm., M.R.C.S., L.S.A: Eng., West Riding Anylum, Wakefield.

Powell, Eran, M.R.C.S. Enq., L.S.A., Senior Assistant Medical Officer, Kent County Asylum, Barming Heath.

Prichard, Thomas, M.D. Glas., M.R.C.P. Lond., F.R.C.P. Edin., late Modical Sunerintendent, Glas. Royal Asylum; Abington Abbey, Northampton.

Pringle, H. T., M.D. Glasg., Medical Supt. County Asylum, Bridgend, Glamorgan. Esplanade. Sunder'and.

Ray, Isaac, M.D., late Physician to the Butler Hospital for the Insane, Providence; Rhode Island, U.S. (Honorary Member.)

Rayner, Henry, M.D. Aber., M.R.C.S. Eng.) L.S.A., Medical Superintendent, County Asylum, Hanwoll, Middlesex. (Honorary Gen. Secretary.)

Bice, Hon. W. Spring, late Secretary to the Commiseioners in Lunacy.) (Honorary Member.)

Richardson, B. W., M.D. St. And., F.R.S., 12, Hinde Street, W. (Honorary Member.)

Robertson, A., M.D., Medical Superintendent Ararat Asylum, Victoria, Anstralia.

Robertson, Alexander, M.D. Edin., Medical Superintendent, Town's Hospital and City Parochial Asylum, Glasgow.

Robertson, Clarles A. Lockhart, M.D. Cantab., F.R.C.P. Lond., F.R.C.P. Edin., Lord Chancellor's Visitor, 45, Lincoln's-Inn-Fields, London. (General Secretary, 1865.62.) (Editor of Journal, 1862-70.) (PREsIDENT, 1867.) (Honorary Member.)

Robertson, John Charles G., Esq., L.R.C.P. Edin., M.R.C.S. Eng., L.S.A. Lond., late Assistant Medical Officer, County Asylum, Hanwell ; Medical Superintendent, County Cavan District Asylum, Monaghan, Ireland.

Rogers, Edward Conlton, M.R.C S. Eng., L.S.A., Senior Assistant Medical Officer 'I hree Cos. Asylum, Stotfold, Baldoek, Herte.

Rogers, Thomas Lawes, M.D. St. And., M.R.C.P. Lond., M.R.C.S. Eng., Medical Superintendent, County Asylum, Rainhill, Lancashire. (Przsinent, 1874.)

Rorie, James, M.D. Edin., L R.C.S. Edin., Medical Superintendent, Royal Asylum, Dundee. (Late Honorary Secretary for Scotland.)

Raseell, A. P.. M B Ed.. Med Sunt. L ncoln Lunatio Hospital, Lincoln.

Rutherford, Jas., M.D. Edin., F.R.C.P.Edin., late Medical Superintendent, Argyll and Bute District Asylum, Lochgilphead; Medical Superintendent Barony Paroch Asylum, Lensie, near Glaspow. (Hon. Secretary for Scotland.)

Sabben, James, M.D. Edin., Gavendiph House, Ramegate.

Salomon, Ernst, M.D., Medical Superintendent, Malmo Asylum, Eweden.

Sanderson, Alezander, F.R.C.S. Edin., 19 Bridge Street, Musselburgh.

Sankey, R. Heurtley H., M.R.C.S. Eng, Medical Superintendent, Oxford County Asylum, Littlemore, Oxford.

Sankey W. H. O.tavins, M.D. F.R.C.P. Lond. - late Medical Superintendent, Hanwell, Middlesex; Sandiywell Park, Cheltenham, and Almond's Hotel, Clifford Street, Bond Street. (PRESIDENT, 1868.)

Banders, W. Rutherford M D, F.R.C.P Ed., Professor of Pathology, Onir. of Edinburgh, 30 Chnrlntte Square, Edinburgh.

Saundby. Robert, M.B and C.M. Edin, General Hospit al, Birmingham.

Saulle, M. Legrand du M.D. Paris, 9, Boulevard de Sebastopol, Paris. (Honorary Member.)

Saunders, George James S., M.B. Lond., M.R.C.S. Eng., Medical Superintendent, County Asylum, Exminster, Devon.

Savage, G. H.. M.D. Lond., Resident Yhysician, Bethlem Royal Hospital, London. (Editor of Joumal.)

Schlager, L., M.D., Professor of Psychiatrie, 2, Universitäts Platz, Vienna. (Uonorary Mernber.)

Schofield, Frank, M.D. St. And., M.R.C.S.. Camberwell House, Camberwell.

Seaton, Joseph, M.D. St. And., F.R.C.P. Edin., Halliford House, Sunbury.

Seymorr, F., M.R.C.S. Eng. L.S.A. Assist. Med. Officer, Thorpe Asylum, Norwich.

Shaw, Thomas C., M.D. Lond , M.R.C.P. Lond., Medical Superintendent, Middlesex County Asylum. Ranstead, Surrey,

Sheaf, C. A. F., M.R.C.P., F.R.C.S.E , Surgeon to the North Lonsdale Hospital, Barrow-in Furness, and Harting ${ }^{\dagger}$ on Street, Barrow in Furness, Lancashire.

Sheppard, Edgar, M.D. St. And., M.R.C.P. Lond., F.R.C.S. Eng., Medical Superintendent, County Asylum, Colney Hatch, Middlesex.

Sherlock, James, M.D., Edin., M.R.C.P. Lond., F.R.C.S. Edin., Medical Superintendent, County Asylum, Powick, Worcester.

Shuttlewortb, G. E., M.R.C.S. and L.8 A. Engl., Medical Superintendent, Royal Albert Asyloun, Lancaster

Sibbald, John, M.D. Edin., F R C.P. Ed., M.R.C.S. Knq., Commissioner in Lunacy for Scotland, 8, Bt. Margaret's Road, Edinburgh. (Editor of Journal 1871-72.)

Simpson, Alexander, M.D., Professor of Midwifery, University, Edinburgh, 62,

Bkae,'C. H., M.D. St. And., Medical Superintendent, Ayrehire District Asylum, $\Delta$ yrahire, Glenga.l, Ayr. 
Skae, F. W. A., M.D. St. And., L.R.O.S Edin., Medical Inspeotor of Asylums, New Zealand, Wellington, N.Z. (Hon. Becretary for Scotland, 1872-76.)

Smart, Andrew, M.'. Edin., F.R.C.P. Edin., 24, Melville Street Edinburgh. Smith Patrick, M.A. Aberdeen, M.D. Dydney, New South Wales, B

Smith, Frederick Moore, M.D. St. And., M.R.C.S. Eng., late Assistant-Surgeon, 4th Reg.; Hadham Palace, Ware, Herts.

Smith, Robert, M.D. Aber., L.R.O.S. Edin., Modioal Superintendent, County

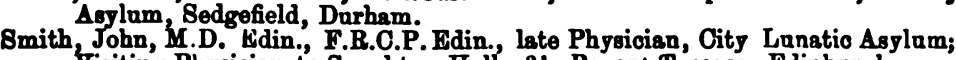
Vieiting Phyeician to Saughton Hall, 31. Regent Turraos, Edinburgh.

Spence, James B., M.D. Ire., Assistant Med. Officer, Earlowood Asylum, Surreg.

Bpencer, Robert, M.R.C.8. Ing., Mod. Superintendent, Kent County Asylum, Chartham, near Canterbary.

Stabb, H., M.D. Medical Superintendent, The Asylum, St. John's, Newfoundland.

Stanley, Hans Sloane, late Chairman of Visiting Magistrates, County Asylum, Hampshire; Paultons, Romsey. (Honorary Mamber.)

Stewart, T. Grainger, M.D., F.R.C.P. Edin., Professor of the Practice of Physic, Univ. of Edinburgh, 19, Charlotte Square, Edinburgh.

Stewart, Henry H., M.D.' Edin., F.R.C.S. Ireland, 71, Eocles St., Dublin.

Stewart, James, B.A. Queen's Üniv. L.R.C.P. Edin., L.R.C.S. Ireland, late Assistant Medical Officer, Kent County Asylum, Maidstone, Mount Hope, Sneyd Park, Briatol.

Stillwell. Henry, M.D. Edin., M.R.C.S. Eng., Mooreroft House, Hillingdon, Middlesex.

Btocker, Alonzo Henry, M.D. St.And., M.R.C.P. Lond., M.R.C.S. Eng., Medical Superintendent, Peckham House Asylum, Peckham.

Strange, Arthur, M.D. Edin., Medical Superintendent Salop and Montgomery Asylum, Bicton, near Shrewrbury.

Sutherland, Henry, M.D. Oron, M.R.C.P. London, 6, Richmond Terrace, Whitehall, 8 W.; Blacklands Honse, Chelses ; and Otto House, Hammersmith.

Sutton, H. G., M.D. Lond., F.R.C.P., Physician to the London Hospital, 9, Hinsbury Square, E.C.

Swain, Edward, Esq.,M.R.C.S., Medical Superintendent, Three Counties' Asylum, Stotfold Baldock.

Swanson, George J., M.D. Edin., Lawrence House, York.

Tate, William Barney, M.D. Aber., M.R.C.P. Lond., M.R.C.S. Eng., Medical Superintendent of the Lanatic Hospital, The Coppice, Nottingham.

Terry, John, M.R.C.S. Eng. Bailbrook House, Bath

Thompson, George, L.R.C.P., M.R.C,8., Medical Superintendent, Bristol Lunatic Asylum, Stapleton.

Thomson, William A., F.K.C.S.I. (Eram.), late Assistant Medical Officer, Kent Lanatic Asylum, Chartham Down; and Wembley, Budbury, Midulosex.

Thurnam, Francis Wyatt, M.B. E.din.. C.M.. 22, Cotham Row, Bristol.

Toller, Ebenezer, M.R.C.S. Eng., late Medical Superintendent, St. Lake's Hospital ; Medical Superintendent, County Asylum, Wotton, Gloucesterehire.

Townsend, Charles Peroj, M.R.C.S. Eng., Assist. Med. Oficer, Barnnood House, Gloucester.

Tuke, John Batty, M.D. Edin., 20, Charlotte Square, Edinburgh. (Honorary Secretary for Scotland, 1869-72.)

Tuke, Daniel Hack, M.D. Heidel., F.R.C.P. Lond., M.R.C.S. Eng., late Visiting Physician, the Retreat, York; 5, Charlotte street, Bedford aquare, London. (Editor of Journal.)

Tuke, Thomas Harrington, M.D. St. And. F.R.C.P. Lond. and Edin., M.R C.8. Eng. Visiting Physician, Northumberland House, Stoke New. ington; 37, Albemarle Street, and The Manor Houee, Chiowiak. (General Secretary, 1862-72.) (President, 1873.)

Turner, Thomas, M.D., L.R.C.P. Edin., M.R.C.S. Eng., Northumberland House, Stoke Newing on.

Turnball, Adam Rohert, M.B., C.M., Edin., Assist. Physician, Buyal Edinburgh

Tweedie, Alexander M.D. Edin. F.R.C.P. London, F.R.S., late Framiner in Medicine, University of London, Visiting Physician, Northumberland House, Stoke Newington, 119, Pall Mall, and Bute Lodge, Twickenham. (Honorary Member.)

Tyner, George St. G., L.K. and Q.C.P. Ireland, L.B.C.S. Ireland, Recident Physician, Downpatrick Distriot Hospital for the Insane.

Urquhart, Alexr. Beid, M.B. O.M.. Ticehurst, Bussex.

Wade, Arthur Law, B.A., M.D. Dab., Senior Asoistant Medioal Officer, Warwick County Asplam, Hatton, Warwiokshire.

Walford, Ed ward, M.B.C.S. and L.S.A. Eng., 2, Paragon, Ramsgate.

Wallace, James, M.D., Modical Superintendent, Greonock Parochial Aeglum, Groenock.

Wallis, John A., M.B. Aberd., L.B.C.P. Edin., Medical Superintendent, Boro' $\Delta$ sylum, Kingston-upon-Hull 
Walsh, Francis !Delaval, M.R.C.S. Edin., late Medical Superintendent, Lanstio Hospital, Lincoln, M anor House, Heighington, Lincoln.

Ward, Frederic H., M.R.C.S. Eng., L.S.A., Assistant Medical Officer, County Asylum, Tooting, Surrey.

Ward, J. Bywater, B.A., M.B. Cant., M.R.C.S. Eng., Medical Superintendent, Warneford Anylum, Oxford.

Warwick, John, F.R.C S. Eng., 25, Woburn Square, W.C.

Watson, Sir Thomas, Bart , late President of the Royal Coliege of Physicians, M.D. Cantab., D.C.L. Oxon., F.R C.P. Lond., F.R.S., Physician Extraordinary to the Queen, 1'i. Henrietta Street, Cavendish Square (Honorary M ember).

West, Francis John, M.R C.S. Eng., Medical Superintendent, District Asylum, Omagh, Tyrone.

Westphal, C. Professor, 40, Manerstrasse, Berlin. (Honorary Member.)

Whitcombe, Edmund Banks, Esq., M.R.C,S., Borough Lunatic Asylum, Birmingham. Wickham, R. H. B., F.R.C.S. Edin., Medical Superintendent, Borough Lunatio Asylum, Newcastle-on-Tyne.

Wilkinson, Matthew Eason, M.D. Edin. F.R.C.P. Phrsician to the Manchester Royal Infirmary and Lunatic Hospital, 25, Gore St., Greenheys, Manchester. Wilks, Samuel, M.D. Lond., F.R.C.P. Lond., Physician to Guy's Hospital; 77, Grosvenor Street, Grosve or Square.

Wilkes, James, F.R.C.S. Eng., late Commissioner in Lunacy; 18, Queen's Gardens, Hyde Park. (Honorary Member.)

Willett Edmund Sparshall, M.D. St. And. M.R.C.P. Lond. M.R.C.S. Eng Wyke House, Sion Hill, Isleworth, Middlesex; and 4, Suffolk Place, Pall Mall.

Williams, Llewellyn, M.D. Edin., M.R.C.P. Lond., L.R.C.S. Edin., 9, Leonard Place, Kensington, $\mathrm{W}$

Williams, S. W. Duckworth, M.D. St. And., L.R.C.P. Lond., Medical Superintendent, Sussex County Asylum, Haywards Heath.

Williams, W. Rhys, M.D. St And., M.R.C.P. Ed., F.K. and Q.C.P., Ire. (Hon. Mem( er), late Resident Physician, Bethlem Hospital, London; Com. missi ner in Lunacy. 19, Whitehall Place.

Williams, William. M.B. Lond., Medioal Superintendent, North Wales County Lunatic Asylum, Denbigh.

Williams, Willism White, M.D. St. And., F.R.C.P. Lond., Consulting Physician, County Asylum, Gloucester; Hayes Lodge, Sydenham Road, Cheltenham. (Hon. Goneral Secretary, 1847-1855.)

Williams, William, M.D., Qu. Univ. Irel., M.R.C.P. Lond., 70, Rodney street, Liverpool.

Wilton, Francis, M.R.C.S. Eng., late Medical Superintendent, Joint Countio Asylum, Carmarthen, Gloucester; Ridgway, Ticehurst, Sussex.

Winn, James M. M.D. Glasg., M.R.C.P. Lond, late resident Physician, Sussex House Asylum; 31, Harley Street, Cavendish Square, W.

Winslow, Henry Forbes, M.D. Lond., M.R.C.P. Lond., Sussex House, Hammersmith, Middlesex.

Winslow, Lyttleton S., M.B.Camb., M.R.C.P. Lond , D.C.L. Oxon, 23, Cavendishsquare, London, W.

Worthington, Thos. Blair, M.A., M.B., and M.C. Trin. Coll., Dublin, Senior Assistant Medical ifficer, County Asylum, Haywards Beath.

Wolf, James de, M.D.Edin., Medical Superintendent, Hospital for Insane, Halifax, Nora Scotia.

Wood, William M.D. St. And., F.R.C.P. Lond., F.R.C.S. Eng., Visiting Physician, St. Luke s Hospital, late Medical Officer, Bethlehem Hospital ; 99, Harlej Street, and The Priory, Roehampton. (President, 1865.)

Wood, Thomas Outterson, M.R.C.P. Edin., F.R.C.8. Edin., M.R.C.S. Engl., Medical snperintendent General Lunatic Asylum. Isle of Man.

Wood, B. T. Esq., M.P., Chairman of the North Riding Asylum, Conyngham Hail, K̇narebboro. (Honorary Member.)

Woods, Oscar T., B.A.M.B. Dub., Medical Superintendent, Asylum. Killarney

Wright, Francis J., M.B. Aberd., M.R.C.S., Eng., Nürthumberland Uouse, Stoko Newington, N.

Wright, John Fred, M.R.C.S. Eng., L.S.A., Asst. Medical Officer, County Asylum, Hanwell, Middlesex.

Wyatt, William H., Esq., J.P., Chairman of Committeo, County Asylum, Colney Hatch, 88, Regent's Park Road. (Honorary Member.)

Yellowlees, David, M.D. Edin., F.F.P.S. Glas., Physician Superintendent, Royal Asylum, Gartnavel, Glasgow.

Younger, E. G., M.R.C.S. Eng., Asst. Medical Officer, County Asylum, Hanwell, Middlesex.

Members are requested to send changes of address or any altoration required to Dr. Rayner. the Honorary Bocretary, County Asylusm, Bamsoell, Middlesex, and in druplicate to the Printer of the Journal, Geo. P. Bacon, Levoes, Buseses. 


\section{Messrs. MACMULIAN \& Co.'s PUBLICATIONS.}

Recently Published in Crown 8vo., Price 68.

INSANITY IN ANCIENT AND MODERN LIFE. WITH CHAPTERS ON ITS PREVENTION. By D. HACK TUKE, M.D.

"A work like this, embodying the matured conchsions of thoughtful study and ertensive obserration, free from dogmatism and exaggeration, from sensational statements and vicious logic, is always weloome. So muoh has appeared on this subject abounding in pernicious errors, that the ralue of \& good book like this can scaroely be over estimated." -The Amorican Journal of the Medical Scionces, July, 1878.

"Dr. Hack Tuke has lately giren to the public another of the books which while interesting and suggestive to the medioal practitioner are highly instructive to the public at large. The present work, like those wo already owe to the same suthor, exhibits deep research in various directions and teams with allusions and quotations which prove the author to be not only an accomplished pasohological physioian, but a scholar of no mesn order. In conclusion, we hare to thank Dr. Tuke for an interesting and suggestive work." - Modical Timos and Garette, June 15, 1878.

HABITUAL DRUNKENNESS AND INSANE DRUNKARDS. By J. C. BUCKNILL, M.D., F.R.S. Crown 8ro., 2s. 6d. (Just Published).

Works by Professor Maudsley, M.D.

THE PHYSIOLOGY OF MJND. Being the first part of a Third Edition, revised, enlarged, and in great part re-written, of "The Physiology and Pathology of Mind." Crown 8vo., 10s. 6d.

BODY AND MIND : AN ENQUIRY INTO THEIR CONNECTION AND MUTUAL INFLUENCE SPECIALLY WITH REFERENCE TO MENTAL DISORDERS. Second Edition, enlarged and revised, with Psychological Essays added. Crown 8ro., 6s. 6d.

Quarterly, price 2s. 6d.

BRAIN : A Journal of Neurology. Edited by Drs. BUCKNILL, CRICHTON.BROWNE, FERRIER, AND HUGHLINGSJACKSON.

Contents :-Original Articles, Clinical Cases, Oritical Digests and Notices of Books, Abstracts of British and Foreign Journals.

[Part III. in October.

Price 1s. 6d. Monthly.

THE PRACTITIONER : a Journal of Therapeutics and Public Health. Edited by T. LAUDER BRUNTON, M.D., F.R.S.

Contents :-Original Communications, Reviews, Clinic of the Month, Extracts from British and Foreign Journals, Notes and Queries, and the Department of Public Health.

MACMILLAN \& CO., LONDON. 
With Engravings, 8v0., I4s.

\title{
ON IDIOCY AND IMBECILITY.
}

\author{
By WILliaM W. IRELAND, M.D.,
}

Medical Superintendent of the Scottish National. Institution for Imbecilo Children at Larbert.

\section{Conterts.}

1. Definition of Idiocy and Imbecility

2. Statistics of Idiocy

3. Causes of Idiocy

4. Classification of Idiocy

5. Genetous Idiocy

6. Microcephalic Idiocy

7. Eclampsic Idiocy

8. Epileptic Idiocy

9. Hydrocephalic Idiocy

10. Paralytic Idiocy

11. Cretinism

12. Traumatic Idiocy

13. Inflammatory Idiocy

14. Idiocy by Deprivation
15. Growth of Idiots, and other Miscellaneous Matters

16. Insanity in Children, and Insane Idiots and Imbeciles

17. Sensory and Mental Deficiencies of Idiots

18. Best Methods of Educating Idiots and Imbeciles

19. Laws for Idiots and Imbeciles

20. Wolf Boys

21. List of Training Schools for Idiots and Imbeciles

Appendix

"It is difficult in the limits of a review to do justice to its many excellencies. It must be read to be appreciated."-British Medical Journal.

"We can cordially recommend the work. . . . Readers will find it to be a pleasant and interesting book on a repulsive and uninteresting subject."Practitioner.

J. \& A. ChURCHILL, New Burlington Street.

Crown 8vo., 5s.

A MANUAL OF NECROSCOPY : OR, A GUIDE TO POST MORTEM EXAMINATIONS, with Notes on the Morbid Appearances, and Suggestions for Medico-Legal Examinations.

BY A. H. NEWTH, M.D.

“This Manual may be confidently recommended, and will, we are inclined to think, supply a want felt by the general body of the profeselon." - Lancet.

"Embraces in its scope a systematic arrangement of the lesions which may be mot with in post mortom investigations. Considering the restricted limits within which the suthor has apportioned his task, we investigations, Considering the restricted limits within which the author han apporti
consider that he has done his work thoroughly and well."-Lomion I Idical Record.

"This very useful book supplies a well recognized want. The author treats in a singularis able and lucid manner of those points which a student or practitioner requires to have at his finger ends in the Post-mortem Room. Mnch ralnable information is found in all parts of the book, which is exactly the thing for all who are purening any pathological work." -Guy's Hoppital Garette.

SMITH, ELDFR \& OO. 1878. 


\section{CONTEATSS OF No. 105.-APRIL, 1878 .}

PART I.-ORIGINAL ARTICLES.

Devid Nicolson, M.D.-The Measure of Jndividual and Social Responsibility in Criminal Cases. W. Julius Mickle, M.D.-The Varieties of General Purulysis of the Insane.

W. Lander Lindsay, M.D. - Spurlous Hydrophotia in Man. (Conciusion.)

Frederiok Treves, M.R.C.s.-The Physiology of Sorde Phases of the Poetic Mtind.

A. H. Newth, M.D. -The Electro-Neural Pathology of Insunity.

County Government Bill.-By an English Medical Superintendent.

Clinical Notes and Cases, - Case of Epilepey, involving the question of Criminul Responxibility; by G. Mackenzie Brcon, M A, M.D.- Notes of A Case in which Hallueinations of four of the Special senses were present-Recovery; by A. I. Turnbull, M.B.

Occasional Notes of the Quarter.-Detention of a Sane Engishwoman for five years in a French Asylum.-Prufessor Tyndall on Consciousness and Organiation, Free Will, \&c.-Mir. PART II.-REVIEWS.

Lecons sur l'Histologie du Système Nervenx. Per L. Raxvien,

Modern Phllosophy, from Deseartes to Selopenhauer and Hartmann. By Fnascis Bowzs, A.M.

Lectures on Diseascs of the Nervous System. By Susrczi. Wriks, M.D.

Fat and Blood, and how to make them. By S. Wrir Miteikn, M.D.

The First and Second Annual heports of the Conmittee for Clapton and Dareith Asylum for Imbeclle Clilitren.

PART III.-PSYCHOLOGICAL RETROSPECT.

1. German Retrospect. By W. W. Ireland, M.D.

2. Freneh Retrospect. By J. G. McDowall, M.B.

PART IV.-NOTES AND NEWS.

The County Financial Boari:s' Bill.-Deputation to the Locul Government Board.-Quarterly Mleetingof the Medico-Psychological Assecintion, at Eninburyh, on Fridav, November 2.-Dr. Lockhar Robertson on County Lunacy Administration. - Tearless Sadness. - Univeraity Lectures ca Iental Diseases.-The late Mir. G. P. Bacon.- Appointments

\section{CONTENTS OF No. 106.-JULY, 1878.}

\section{PART 1.-ORIGINAL ARTICLES.}

Dr. A. Enlenburg and Dr. P. Guttmenn.- Physiology and Puthology of the Sympathetic System of Nerves. Translated by A. NAFIER, M.D.

William W. Ireland, M.D. - On Thought without Words, and the Relation of Words to Thought. Alexander Robertson, M.D. - Observations on Some Points in Cerebral Pathology and on Percussition of the Skull.

Frederiok Treves, M.R.C.S.-The Pligsiology of Some Plinses of the Poetic altad. (Conelusion.) G. Hunter Mackenzle, M.D. - On some Indications for the Use of Digitalis in Acute Mental
Disorders. David Nicolson, M.D.-The Measure of Individusl and Social Responslbility in Criminal Cases.
(Conclusion.,

Chinfeal Notes and Cases.-An Account of Two Cases of Locomotor Ataxin, with Mental Symptoms simulating those of General Paralysis; by J.W. PLAxros, M.R.C.S.

Oocagfonal Notes of the Quarter.- The Report of the Lunney Law Committee of the House of Coinmons, 1878. - Sir James Coxe on Lumucy and its Relations to the State. - On Metalloscopta."Feeding v. Fasting." - Dr. Fraser on the Disadivantages of Boarding out certain Harmless in Asylams,-Spitzkn on Heform in the Selentife Study of Puychiatry.

\section{PART II.-REVIEWS.}

Cyclopadia of the Practice of atedicine. Edited by Dr. H. Vox ZrEarses, Vol. xiv.-Disenses of the

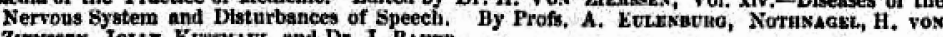
Zisurseses, Jott., Kussiratr., and Dr, J. Batzh.

Insanity in Ancient and Modern Life, with chapters on its Prevention. By Daxige. Hack Teks, M.D.

Gedanken tiber die Socialwissenchaft der Zukunft von I aul Lillenfeld. Dritter Theil: Die Bocinle Paychophysik, Mitau, 1877.1

PART III.-PSYCHOLOGICAL RETROSPECT.

Itallan Paychological Literature. By J. R. (s Aseust, M.D. PART IV.-NOTES AND NEWS.

Quarterly Meeting of the Medlco-Psychological Association, hela at Glasgow on Friday, March 15. 1878. - Retirement of $\$ r$. James Wilkes from the Commission in Lunacy.-Appointment of Dr. W. Rhys Wiliams as Commissioner in Lunacy,-Lunacy Clu tusce of the Sew Stedical Ameniment

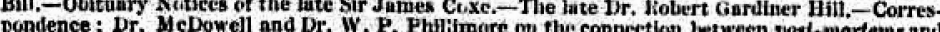
pondence: Dr. MeDowenl nnd Dr. W. P. Philimore on the connection between posi-mortems and Lryser Medico-Pyychologicai Association, se., se. 


\section{JOURNAL OF MENTAL SCIENCE. OCTOBER, 1878.}

CONTFNTS.

PART I,-ORIGINAL ARTICLES.

J. Crichton Browne, M.D. - Presidential Ad Jress delivered at the Royal College of Physiciaus, July 26, $187 \mathrm{~s}$.

Dr. A. Eulenburg and Dr. P. Guttmann.-Physiology and Pathology of the Sympathetic System of Nerves. Translated by A. Napisk, M.D. Part II. William W. Ireland, M.D. - On Thought withont Words, and the Relation of Words to Thought. ((Continuation)

G. E. Shuttleworth, M.D.-A Case of Microcephalic Imbecility, with remarks.

D. Hack Tuke, Mr.D.-A few Notes on Lunacy in Franes, soggested by a recent visit to Freach Asylums.

D. Yellowlees, M.D.-Hnicide by a Somnambulist.

Clinical Notes and Cases.-Case of Malformation of Genitalia with Insanity; by G. H. SAVAgE, M.D. - Notes of a Case of Delusional Insanity, with an account of the Autopsy; by ROB $\triangle R$ S BAKER, M.D.

PART II.-REVIEWS.

Proceedings of the International Congress of Mental Medicine, Paris, August, 1878 .

Friendly Talk with a New Patient-Visiting Day at the Asylum-Work in the Wards by Asylum Attendants. By Kev, H. Hawkiss.

De quelques accid-nts de l'epilepsio et de l'hystéro-épilepsie. Yar EaruLz BoviLi, M D.

A Manual of Necroseopy, or a Guide to the Performance of Pest-Mortem Examinations. By A. H. NEwTH, M.D.

Cyclopædia of the Practice of Medicine. Vol, xiv.: Disenses of the Nervous System and Disturbances of Speech. By Professors A. EuLrenburg,

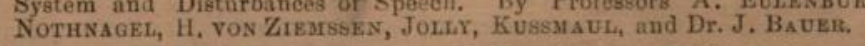

Die Geistesstörungen der Schwangeren, Wöclinerinnen und Säugenden. A Monograph, by Dr. RIPring.

PART III.-PSYCHOLOGICAL RETROSPECT.

1. Ameriean Paychological Literature. By D. Hick Tuks, M.D.

$$
\text { PART IV.-NOTES AND NEWS. }
$$

Thirty-third Annual Meeting of the Medico Psychological Association, held on Friday, July 26.-A ppointment of $\mathrm{D}$. John sibbald as Commissioner in Lunacy for Seotlund - The late Dr. Thomas Howden.-The Law of the fature in regard to the Responsibility of the Insane. -Withdrawal of the County Administration Bill.-Habitual Drunkards Bill-A Wolf Child.Recovery from lnsanity after ten years.-Correspondence-Appointments. -List of Afembers, \&e.

The Editors do not hold themselves responsible for the vieus of Contributors whose names are signed.

No. CVIII. (Neio Series, No. 72) will be published on the 1 st of January, 1879. 